\title{
LOW ENERGY IPL THERAPY FOR THE MANAGEMENT OF RECALCITRANT POST- HERPETIC NEURALGIA
}

\author{
*Sherif S. Awad M.D., Ph.D., and Wael Hosam El-Din M.B.B.Ch., M.S.C., M.D. \\ Department of Dermatology, Faculty of Medicine, Minia University, Minia, Egypt.
}

\begin{abstract}
Herpes zoster is a common health problem particularly for the elderly. The pain persisting after the zosteriform lesions have resolved is termed post-herpetic neuralgia (PHN) and is considered a very common and extremely painful, debilitating complication. There are several approaches available to manage PHN, yet persistence of the pain is a very common vexing problem. Light therapy and in particular low-level laser/light therapy (LLLT) is increasingly applied nowadays. It has been suggested that LLLT is possibly beneficial in the management of many different medical conditions, including pain, wound healing and nerve injury. The present study evaluates the application of low incident energy intense pulsed light (IPL) as a possible photobiomodulative approach that can enhance healing and bring relief pain in recalcitrant cases of PHN in elderly patients.
\end{abstract}

Key Words: Post-Herpetic Neuralgia, IPL, light therapy, LLLT.

\section{Introduction}

Herpes zoster is a well known health problem that results from recrudescence of latent varicella zoster virus particles from the dorsal root or cranial nerve ganglia, which is present since any previous primary infection with varicella (chicken pox) ${ }^{(1)}$.

After the primary infection the virus is often reactivated, but competent cell mediated immunity prevents clinical disease. The commonest cause of viral recrudescence is a decline in cell-mediated immunity related to age. Not only aging, but reduced immunity associated with some malignancies (such as lymphoma), treatment of malignancy (chemotherapy or radiotherapy), HIV infection, and use of immunosuppressant drugs after organ transplant surgery or for disease management (such as steroids) are also risk factors for the occurrence of herpes zoster ${ }^{(2)}$.

\section{Post-Herpetic Pain}

The pain persisting after herpes zoster has run its course, referred to as post-herpetic neuralgia (PHN), is the commonest and most feared complication. Post-

\section{Addressee for Correspondence:}

Sherif S. Awad, MD, PhD.

8, Abdel-Menam Garby Square, Minia, 61111, Egypt.

Telephone : +20127340233

Email : sherifu@rocketmail.com herpetic neuralgia is characterized as a continuous, lancinating pain and allodynia that has the potential to begin after healing of the zosterform rash and persists for months or years, and possibly throughout life $(3,4)$. The pain and hypersensitivity mat be so severe that affected subjects cannot even bear contact with clothing.

The most well established predicting factors for PHN include increasing age, greater severity of acute pain during the zoster arttack, more severe rash, female sex and a prodrome of dermatomal pain before the onset of the rash. Patients with all of these risk factors may have as much as a $75 \%$ risk of persisting pain six months after the rash onset. However, the accuracy of these predictors varies among studies and none seems especially helpful for planning early and effective prevention of $\mathrm{PHN}^{(2,4)}$.

Other possibilities for predicting PHN include biochemical markers in the cerebrospinal fluid. The concentration of IL-8 in cerebrospinal fluid correlates with the degree and duration of $\mathrm{PHN}(4,5)$.

Patients over the age of 50, irrespective of other risk factors, are at much greater risk of developing PHN and should be offered early treatment inhibiting replication of the varicella zoster virus. The antiviral agents acyclovir, famciclovir, and valaciclovir attenuate

Manuscript received: December 2007

Accepted for publication: December 2007 
the severity of zoster: specifically, the duration of viral shedding is decreased, rash healing is hastened, and the severity and duration of acute pain could be reduced. Unfortunately, despite treatment, at least 20\% of elderly patients, continue to report pain up to and even well beyond six months after the onset of the rash (2). Established post-herpetic neuralgia may be intractable and lead to considerable disability and suffering in the elderly patient's final years of life (2).

First line therapy includes attention to psychosocial factors as well as medical treatment. Patients need clear advice on measures such as the use of natural fibre clothing and maintenance of social and physical activities. If appropriate dosages of a tricyclic antidepressant (preferably nortriptyline) or gabapentin do not provide adequate relief, strong opioids such as oxycodone, morphine, or methadone may be required. Combination therapy is common, and the multiple putative mechanisms of neuropathic pain provide a rationale for this approach, but studies have not been conducted to examine its efficacy. All these medications should be used with close monitoring as they are associated with considerable risks in elderly patients $(2$, 6).

A local anaesthetic (lidocaine 5\%) patch can provide pain relief with minimal side effects and, where it is available, is often used in combination with oral medications. Some patients can benefit from other techniques, including topical capsaicin and topical application of aspirin suspended in a volatile substance such as acetone. A highly controversial therapy for patients failing to respond to conventional management involves intrathecal injection of methylprednisolone. Although results seemed good and complications few, the risk of serious complications may preclude use of this treatment until well designed studies have confirmed efficacy and lack of morbidity $(2,5)$.

Early treatment in post-herpetic neuralgia may be more effective than later treatment ${ }^{(7)}$. Although additional research is needed to confirm this, it is clearly desirable to relieve chronic pain as early in its course as possible. The primary care provider can often achieve effective pain control in patients with either herpes zoster or post-herpetic neuralgia. However, when either the acute pain of herpes zoster or the chronic pain of post-herpetic neuralgia is not rapidly and effectively controlled in primary care with the first line medications, referral to a pain specialist or pain management centre can be considered to reduce pain and to improve the patient's quality of life ${ }^{(2)}$.

\section{Light Therapy}

Nowadays sources of light are widely used in therapy. They have been adapted to many medical procedures ranging from surgery, oncology, physiotherapy, dentistry, dermatology and photobiomodulation (8)

Low-level laser/light therapy (LLLT) is increasingly used nowadays. It has been suggested that LLLT may be beneficial in the management of many different medical conditions, including pain, wound healing and nerve injury (9-11). Because of the low power levels used the possibility of sensory nerve stimulation via the phenomenon of shock or heat is excluded. Thus senseless optical stimulation is achieved. The non-invasive nature of light biomodulation has made its sources an attractive alternative in medical acupuncture in recent years (8).

The exact mechanisms by which LLLT facilitates healing \& pain relief are gradually being elucidated, but many remain poorly understood. In vitro studies have shown an increase in fibroblast proliferation after irradiation, suggesting that LLLT therapy may facilitate fibroplasia during the repair phase of tissue healing (9). Other data suggest that LLLT facilitates collagen synthesis, keratinocyte cell motility, and growth factor release and transforms fibroblasts to myofibroblasts (10,12-14).

LLLT may also cause release of tissue growth factors into the circulation, which may affect surrounding tissues or entire systems. Indirect healing could be a very beneficial effect in treating tissue damage of large size or at multiple locations. It might also suggest that deeper tissues could be affected by light therapy (9).

Indication for light therapy for pain management has included various kinds of diseases such as herpes zoster, post herpetic neuralgia, cervical pain, lumbago due to muscle contracture, complex regional pain syndrome, arthralgia, rheumatoid pain etc. $(15,16,17)$

Recent literature has provided examples where enhancement of neural repair and regeneration of neural tissue with increased axonal density has been proved after light treatment $(18,19,20,21)$. Angiogenesis is also a recorded effect of LLLT, and this should have a main role in enhancing healing and neural regeneration (22). Irradiation with low incident levels of light energy can therefore be considered as a useful noninvasive adjunct to promote neuronal healing.

Application of phototherapy in the management of post-herpetic neuralgia has been described in the literature for many years. Light therapy using UVB or UVA proved to be useful in reducing the incidence and severity of PHN. The healing effects were not restricted to the treated lesions and were believed to 
involve systemic transmission of light-mediated biological energy throughout the body (23, 24).

In this study, we attempted to evaluate the application of intense pulsed light (IPL) as a possible biomodulatior through treatment with low incident levels of non-coherent polychromatic light energy with the aim of facilitating the management of recalcitrant postherpetic pain in elderly patients.

\section{Patients and methods}

Twelve male patients more than 50 years of age suffering from persistent post-herpetic pain, following trunkal zoster, were candidates for the study (age range, 55 - 76 yrs, mean $5.41 \pm 6.24 \mathrm{yrs}$ ). All patients had received acyclovir $800 \mathrm{mg} / 5$ hourly for 1 week during the active attack. After healing of the skin lesions, herpetic pain was approached in classic ways by prescribing a combination of ketoprofen $200 \mathrm{mg}$ and carbamazapine $400 \mathrm{mg}$ daily for 3 weeks with no appreciable improvements and subsequent failure of the subjects to engage again in their daily activities. Following a full explanation of the trial and the treatment procedure, all patients gave their written informed consent. Approval for the trial was obtained from the Ethics Committee of the Department of Dermatology, Minia University, and the trial was conducted according to the precepts of the Declaration of Helsinki.

The device used during the procedure was the Quanta Light IPL (Quanta system SpA Medical division, Milano, Italy). A $610 \mathrm{~nm}$ cut-off filter was used, allowing polychromatic emission from $610 \mathrm{~nm}$ to $1200 \mathrm{~nm}$. The dose used was $12 \mathrm{~J} / \mathrm{cm}^{2}$, and pulses were delivered singly in with a pulse duration of $40 \mathrm{~ms}$. During treatment, a coupling gel was applied to the target tissue, allowing the treatment head to be held 1-2 mm from the skin. A dermatomal approach was chosen. The handpiece was held stationary and light pulses were delivered, moving along the whole dermatome starting from the dorsal root zone of each vertebra and moving the handpiece laterally away from the spine till the most median painful point ventrally, along the course of the affected nerve. Both the subject and the therapist wore appropriate safety goggles during the treatment. Repetition of sessions was advised every week for one month.

\section{Results}

All patients successfully completed the study. Evaluation of pain relief was assessed each week after undergoing an IPL session, and compared to the pre- ceding pain condition. Improvement and pain relief were estimated according to the patients' own assessments, but a visual analogue scale (VAS) was also utilized to provide a more objective evaluation, rather than only subjective patient evaluation, and to help confirm the latter. The percentage of improvement was described in $10 \%$ blocks.

Percentage of pain relief \& improvement after the $1^{\text {st }}$ session of therapy ranged from $50 \%$ to $80 \%$ with a mean of $64.16 \pm 9 \%$ and was very satisfactory for the patients. Improvement on the following weekly sessions declined, and was ranging only between 0-30\% (Fig. 1). At the end of the study all patients were satisfied with the results and could return to their daily physical activities. No side effects or sequela was reported by any patient during or after the sessions throughout the study.

\section{Discussion}

There are four possible methods of irradiation with light sources reported for pain management; irradiation of acupuncture points, of trigger points, along nerves causing pain and of the stellate ganglion area. In this study we delivered the light therapy along the course of affected nerve trunk guided by the preceding $\mathrm{HZ}$ eruption.

Recent studies have confirmed the efficacy of LLLT phototherapy in healing and improving nerve functions after neural injury leading to significant functional recovery. Wave lengths from 600 to 780 were found to provide best results $(11,20,25)$. We introduced IPL treatment with a dose of $12 \mathrm{~J} / \mathrm{cm}^{2}$ and polychromatic light from $610 \mathrm{~nm}-1200 \mathrm{~nm}$ using a cut-off fil-

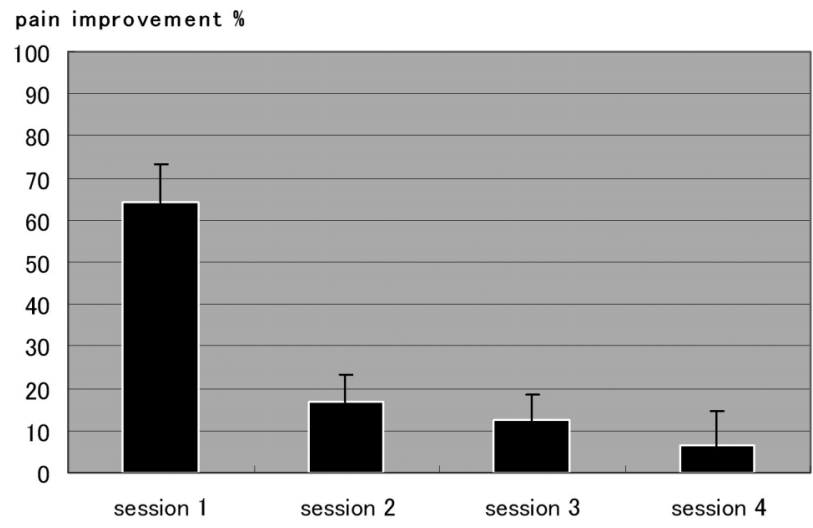

Fig. 1: Average pain relief percentage after each session of IPL. Improvement of pain sensation was achieved after each session of therapy, and was most noticeable after the first session. 
ter. These criteria were decided according to the best and optimum results obtained in previous experimental trials evaluating the LLLT efficacy in pain relief $(9,26$, 27)

Sessions were repeated even after obtaining satisfactory results following the first session following previous reports that multiple doses of LLLT could improve the efficacy. The dose per IPL shot was only $12 \mathrm{~J} / \mathrm{cm}^{2}$ in every session and sessions were repeated weekly $(10,16)$.

Because of the low incident energy levels used, there was no sensory nerve stimulation via the phenomenon of shock or heat. Thus only noninvasive photobiomodulation of the target tissues was achieved. As previously reported, due to the low intensity of the output, the subjects had no thermal or tactile clues as to the actual treatment and remained unaware of the application of the therapy (8). The low intensity IPL dosage used did not result in any erythema or burning sensation at targeted skin, sequelase which are commonly associated with the application of IPL systems for other purposes like hair removal where much higher energy is delivered, when burning sensation and/or pain can be felt by the patients during the procedure. Furthermore, in the present study, no erythema or post-IPL pigmentation resulted during the follow up.

The low incident levels of IPL energy appeared to act through a biomodulatory effect that enhanced neuronal regeneration and healing, possibly through fibroblast proliferation, angiogenesis, release of growth factors and immunological modulation at irradiated site, but remote effects could also be anticipated $(9,10$ $12-14,20,22)$. These factors enhanced healing and regeneration of damaged neurons and subsequently the pain decreased dramatically.

In spite of the dilemma of persistence of pain after oral medications prescribed prior to IPL treatment, a very dramatic improvement, with pain relief exceeding 50\%, was detected after the first session in all patients, and by completion of the sessions all patients were able to resume their regular daily activities.

Coherence, comprising collimation, phase and monochromaticity, is the well-recognized beneficial characteristic of laser beams, which were used previously in LLLT for pain relief, but the IPL system is a device emitting light which is noncoherent, having neither collimation, phase nor monochromaticity. It has been shown that the coherent nature of light was not essential for angiogenesis, a possible enhancer of healing after neural trauma or inflammation ${ }^{(22)}$. So in the present study, the lack of coherence did not change the beneficial outcome: on the contrary we believe that the polychromatic characteristic of IPL energy provided a greater spectral range of photons, possibly resulting in the photobiomodulation of more targets.

Pain reduction rheumatoid patients has been reported as being successfully managed in the literature ${ }^{(15)}$, yet other studies failed to prove the efficacy of LLLT in pain relief in rheumatoid patients as the benefit with an improvement of $72 \%$ was identical in both hands of a patient, one of which underwent only placebo treatment (16). On the other hand, it should always be remembered that bilateral improvement can also be attributed to the expected release of photomediated beneficial growth and other factors into the systemic circulation, thus providing a remote beneficial response to the non-irradiated hand (9). In this way, the $72 \%$ should be considered as appreciable success. With this success, we may also recommend the use of IPL device, set to deliver low incident levels of energy, in managing other types of pain such as rheumatoid pain, and not only post-herpetic neuralgia.

The remote beneficial effect may also explain the correction of central nervous system (CNS) changes that contribute to the persistence of neuropathic pain in PHN. It has been revealed recently that immune activation at the dorsal root ganglion (DRG) or in the dorsal roots themselves, causes hyperalgesia and that the immune system actively participates in creating and maintaining neuropathic pain, the so-called 'kindling' effect. It has also been reported that trafficking of leukocytes into the central nervous system and major histocompatibility complex (MHC) class II cells are associated with mechanical allodynia and persistent neuropathic pain ${ }^{(28)}$.

No side effects or adverse sequelae were recorded in any patients following the use of low incidentenergy IPL. This was expected, as LLLT has no previously reported particularly dangerous effects on the elderly, in contrast with the medications that are usually prescribed to control post-herpetic pain and require close monitoring, posing considerable risks in elderly patients $(2,6)$.

The literature still contains controversial opinions regarding the efficacy of light therapy. After this clinical study, however, we now recommend its use, and value its presence in the armamentarium of the dermatologist for post herpetic neuralgia to overcome this very problematic and potentially intractable symptom after herpes zoster infection in the elderly. Low level light therapy with intense pulsed light as provided us with the solution we were looking for when confronted with many cases of unresponsiveness to the approach indicated by the many classic modalities 
Until further confirmation of its value through more controlled studies, and investigative work to illustrate the exact way by which low level light therapy with intense pulsed light helped this process of pain eradication and neuronal regeneration, we recom-

\section{References}

1: Gnann JW Jr, and Whitley RJ (2002): Herpes zoster. The New England journal of medicine, 347:340-346.

2: Johnson RW, and Dworkin RH (2003): Treatment of herpes zoster and postherpetic neuralgia. British Medical Journal, 326(7392): 748-750.

3: Cunningham AL, and Dworkin RH (2000): The management of post-herpetic Neuralgia. British Medical Journal, 321:778-9

4: Kotani N, Kudo R, Sakurai Y, Sawamura D, Sessler DI, Okada H, Yamagata T, Yasujima M, and Matsuki A (2004): Cerebrospinal fluid IL-8 Concentration and subsequent development of Postherpetic neuralgia. The American Journal of Medicine, 116 (5): 318-324.

5: Kotani N, Kushikata T, Hashimoto H, Kimura F, Muraoka M, and Yodono M (2000): Intrathecal methylprednisolone for intractable postherpetic neuralgia. The New England journal of medicine, 343:1514-1519.

6: Kanazi GE, Johnson RW, and Dworkin RH (2000): Treatment of postherpetic neuralgia: an update. Drugs, 59:1113-1126.

7: Bowsher D (1996): Postherpetic neuralgia and its treatment: a retrospective survey of 191 patients. Journal of pain and symptom management, 12:290-299.

8: Valchinov ES and Pallikarakis NE (2005): Design and testing of low intensity laser biostimulator. Biomedical Engineering Online, 13;4 (1):5.

9: Hopkins J, McLoda TA, Seegmiller JG, and Baxter GD (2004): Low-Level Laser Therapy Facilitates Superficial Wound Healing in Humans: A TripleBlind, Sham-Controlled Study. Journal of atbletic training, 39 (3): 223-229.

10: Khadra M (2005): The effect of low level laser irradiation on implant-tissue interaction. In vivo and in vitro studies. Swedish dental journal Suppl., 172:163.

11: Yasukawa A, Ohrui H, Koyama Y, Nagai M, and Takakuda K (2007): The effect of low reactive-level laser therapy (LLLT) with helium-neon laser on operative wound healing in a rat model. The Journal of veterinary medical science, 69 (8):799- mend it as a valuable alternative for patients with recalcitrant pain who reject injection or patients who are not indicated for nerve block because of the preexistence of contraindications such as bleeding tendency, and so on.

806.

12: Haas AF, Isseroff RR, Wheeland RG, Rood PA, and Graves PJ (1990): Low-energy helium-neon laser irradiation increases the motility of human keratinocytes. Journal of Investigative Dermatology, 94:822-826.

13: Yu W, Naim JO, and Lanzafame RJ (1994): The effects of photo-irradiation on the secretion of TGF and PDGF from fibroblasts in vitro. Lasers in surgery and medicine, 15 (3):281-9.

14: Houreld NN, and Abrahamse H (2008): Laser light influences cellular viability and proliferation in diabetic-wounded fibroblast cells in a dose- and wavelength-dependent manner. Lasers in medical science, 23 (1):11-8.

15: Walker J B, Akhanjee L K, Cooney M M, Goldstein J, Tamoyoshi S, and Segal-Gidon F (1987): Laser therapy for pain of rheumatoid arthritis. The Clinical journal of pain, 3: 54-9.

16: Heussler JK, Hinchey G, Margiotta E, Quinn R, Butler P, Martin J, and Sturgess AD (1993): A double blind randomised trial of low power laser treatment in rheumatoid arthritis. Annals of the Rheumatic Diseases, 52: 703-706.

17: Saeki S (2006): Equipment for low reactive level laser therapy including that for light therapy. Masui. The Japanese journal of anesthesiology. 55 (9):1104-11.

18: Miloro M, Halkias LE, Mallery S, Travers S, and Rashid RG (2002): Low-level laser effect on neural regeneration in Gore-Tex tubes. Oral surgery, oral medicine, oral pathology, oral radiology, and endodontics, 93 (1): 27-34.

19: Gigo-Benato D, Geuna S, and Rochkind S (2005): Phototherapy for enhancing peripheral nerve repair: A review of the literature. Muscle \& Nerve; 31, $694-701$

20: Rochkind S, Drory V, Alon M, Nissan M, and Ouaknine GE (2007): Laser Phototherapy (780 $\mathrm{nm})$, a New Modality in Treatment of Long-Term Incomplete Peripheral Nerve Injury: A Randomized Double-Blind Placebo-Controlled Study. Photomedicine and Laser Surgery, 25 (5): 436-442.

21: Rochkind S, Leider-Trejo L, Nissan M,. Shamir MH, 
Kharenko O, and Alon M (2007): Efficacy of 780nm Laser Phototherapy on Peripheral Nerve Regeneration after Neurotube Reconstruction Procedure (Double-Blind Randomized Study). Photomedicine and Laser Surgery, 25 (3): 137-143.

22: Corazza AV, Jorge J, Kurachi C, and Bagnato VS (2007): Photobiomodulation on the angiogenesis of skin wounds in rats using different light sources. Photomedicine and laser surgery, 25 (2):102-6.

23: Martin WJ, and Stoneburner J (2005): Symptomatic relief of herpetic skin lesions utilizing an energybased approach to healing. Experimental $\varepsilon$ Molecular Patbology, 78 (2):131-4.

24: Jalali $\mathrm{MH}$, Ansarin $\mathrm{H}$, and Soltani-Arabshahi $\mathrm{R}$ (2006): Broad-band ultraviolet B phototherapy in zoster patients may reduce the incidence and severity of postherpetic neuralgia. Photodermatology, photoimmunology \& photomedicine, 22 (5):232-7.

25: Gal P, Vidinsky B, Toporcer T, Mokry M, Mozes S,
Longauer F, and Sabo J (2006): Histological Assessment of the Effect of Laser Irradiation on Skin Wound Healing in Rats. Photomedicine and Laser Surgery, 24(4): 480-488.

26: Hawkins D, and Abrahamse H (2007): Influence of broad-spectrum and infrared light in combination with laser irradiation on the proliferation of wounded skin fibroblasts. Photomedicine and Laser Surgery, 25 (3):159-69.

27: Al-Watban FA, Zhang XY, and Andres BL (2007): Low-level laser therapy enhances wound healing in diabetic rats: a comparison of different lasers. Photomedicine and Laser Surgery, 25 (2):72-7.

28: Takeda SM, Takasaki I, Takeda K, Sasaki A, Andoh T, Nojima H, Shiraki K, Kuraishi Y, Hanaoka K, Tokunaga K, and Yabe T (2006): Major Histocompatibility Complex Haplotype Is Associated with Postherpetic Pain in Mice. Anesthesiology, 104:1063-9. 